\title{
Developing an online CPD module on management of breast cancer for general practitioners in Sri Lanka - A need analysis
}

\author{
Thanya I Pathirana ${ }^{1}$, Indika Karunathilake ${ }^{2}$, Sujeewa P.W Palagama ${ }^{1}$, Ishra Nazeer ${ }^{1}$
}

\begin{abstract}
The prevalence of breast cancer in Sri Lanka is on the rise. A need analysis was carried out to identify the learning needs and utilization of currently available learning resources on breast cancer management among the general practitioners (GPs) in Sri Lanka. A pretested questionnaire was posted to 210 GPs affiliated to College of General Practitioners of Sri Lanka. The data were analyzed using descriptive statistics. Response rate was $55.2 \%$. Fifty nine $(50.9 \%)$ participants were aged between $31-50$ years and 77 (66.4\%) were males. Majority $87(75 \%)$ had not previously taken part in any CPD program on breast cancer management. Awareness on the guide for primary care doctors and family physicians on management of breast symptoms $(31,26.7 \%)$ and national guidelines on management of breast carcinoma (20, 17.2\%) were markedly low. Although majority $(99,85.3 \%)$ reported a medium to high involvement in referral, 77(66.4\%) admitted of low involvement in shared follow up care while $72(62.1 \%)$ reported low involvement in palliative care. Accordingly, palliative care $(68,58.6 \%)$ and shared follow up care $(77,66.4 \%)$ were identified to be among the top two priority areas to be included in a future CPD module. Majority $(83,71.5 \%)$ preferred either online or blended modes of learning. In conclusion, participation of GPs in CPD programs on breast cancer management and awareness on current guidelines are deficient. Although current involvement in referral of breast cancer patients for tertiary care is substantial, involvement in shared follow up care and palliative care is low. Thus, a future online CPD module should be established with greater emphasize on these areas.
\end{abstract}

Key words: Continuing Professional development, General Practitioners, Breast Carcinoma

\section{Introduction}

The general practitioners (GPs) have a traditional role in breast cancer management in terms of screening, health promotion and referral.

With enhanced cancer survivorship and more patients seeking services from already overburdened specialist oncology centres, the role of GPs in the continuum of cancer care has significantly evolved in recent times.

${ }^{1}$ PhD student, Center for Research in Evidence Based Practice, Faculty of Health Sciences and Medicine, Bond University, Queensland, Australia.

${ }^{2}$ Director, Medical Education Development and Research Center (MEDARC), Faculty of Medicine, University of Colombo, Sri Lanka.

${ }^{1}$ Surgical Registrar, Post Graduate Institute of Medicine, University of Colombo, Sri Lanka.

${ }^{1}$ Medical Officer, Oncosurgical unit, Teaching Hospital, Karapitiya, Galle, Sri Lanka

Corresponding Author:

Dr Thanya Pathirana, Masters student in Public health, School of Medicine, Griffith University, Queensland, Australia.

Email: thanya.pathirana@griffithuni.edu.au
In developed countries, their roles in shared follow up care with the oncologists and in palliative care within the primary health care setting have been reasonably established (Roorda et al., 2012) and supported by continuing professional development (CPD) training opportunities (Mitchell et al., 2004).

In Sri Lanka, the only training resource available for GPs, apart from the national guidelines, is the "Guide for primary care doctors and family physicians on management of breast symptoms" published by the National Cancer Control Program. This document is not available online [NCCP, 2012). Even though it includes information on the referral pathway and updated screening guidelines, it lacks comprehensive information on novel treatment modalities, shared follow up care and palliative care at primary health care level. Furthermore, it's also worthwhile to note that in Sri Lanka, Elearning has not been widely established so far although it initially aspired to become a significant component of the national CPD program, especially in the peripheral areas of the country (Epa, 2005). 
Thus as an initial step to bridge this critical knowledge gap, an analysis of learning needs of GPs was carried out prior to developing and implementing a novel online CPD module on breast cancer management for general practitioners in Sri Lanka.

\section{Methods}

The need analysis was carried out to identify the learning needs of general practitioners and their awareness and utilization of currently available learning resources on breast cancer management as well as any barriers associated with accessing them. In addition, the level of internet access and computer literacy/competency were also assessed. The data collection instrument was a pretested survey questionnaire which was posted to a randomly selected sample of 210 general practitioners affiliated to College of General Practitioners of Sri Lanka. The data were analyzed using descriptive statistics of SPSS version 20. Ethical approval was obtained from the Ethics review committee, Faculty of Medical sciences, University of Sri Jayewardenapura, Sri Lanka.

\section{Results}

Response rate for the survey was $55.2 \%$. Fifty nine $(50.9 \%)$ participants were in the age range of $31-50$ years and most $(66.4 \%)$ were males. Seventy one (61.2\%) had received postgraduate education out of which $87.9 \%$ had completed it in the field of family medicine. Sixty four general practitioners $(55.2 \%)$ were practicing part time.

Twenty four $(20.7 \%)$ had not undergone general surgical training in internship or postinternship while $79.2 \%$ of them had never taken part in a CPD program on breast cancer management. Overall, $87(75 \%)$ of the total participants had not taken part in any CPD program on breast cancer management previously.

Eighty five (73.3\%) were unaware of the "Guide for primary care doctors and family physicians on Management of Breast Symptoms" published by National Cancer Control Program, while $82.8 \%$ were unaware of national guidelines on management of breast carcinoma.
Although majority (85.3\%) of general practitioners reported a medium to high involvement in referring patients for further care at tertiary level, $66.4 \%$ admitted to their low involvement in shared follow up care while $62.1 \%$ admitted to their low involvement in palliative care.

However, when questioned about priority learning needs that should be addressed in a future CPD module, $58.6 \%$ rated palliative care in breast cancer management to be among the top two priority areas while $66.4 \%$ rated shared follow up care to be among the top two priority areas.

Majority $(71.5 \%)$ preferred either online or blended (online and face to face) modes as their first choices for a future CPD program while $94 \%$ had internet access and $90.6 \%$ reported an average or above competency level in using internet. The characteristics of the respondents are summarised in Table 1, while Table 2 summarizes the reported learning needs.

\section{Discussion}

Response rate for the survey was consistent with previous studies (Smith et al., 2011).

Evidence from developed countries had described higher GP involvement during initial and late stages of the cancer care spectrum which included diagnosis, referral and palliative care (Mitchell et al., 2004; Roorda et al., 2012) while our study showed this involvement was essentially limited to diagnosis and referral for specialist care.

Furthermore, knowledge on palliative care and shared care were chosen to be among top two learning priorities that should be addressed in a future CPD training module on breast cancer management for general practitioners. Thus, it may be proposed that one of the potential factors which could have led to low levels of involvement in palliative and shared follow up care could be due to deficiencies in competence. Studies done elsewhere have pointed out that GPs were keen on taking on the responsibilities of follow up care if the required training was provided to ensure they were equipped with updated knowledge and skills on best practice of managing breast carcinoma at a general practice setting (Grunfeld, 2008; Mitchell et al., 2004). 
Table 1: Characteristics of the Participating General Practitioners

\begin{tabular}{|c|c|}
\hline Characteristic & n (\%) $\quad \mathrm{N}=116$ \\
\hline \multicolumn{2}{|l|}{ Age (years) } \\
\hline$<=30$ & $8(6.9)$ \\
\hline $31-40$ & $32(27.6)$ \\
\hline $41-50$ & 27 (23.3) \\
\hline $51-60$ & $21(18.1)$ \\
\hline$>60$ & $28(24.1)$ \\
\hline \multicolumn{2}{|l|}{ Gender } \\
\hline Male & $77(66.4)$ \\
\hline Female & $39(33.6)$ \\
\hline \multicolumn{2}{|l|}{ Highest level of Professional education } \\
\hline MBBS/MD as first degree & $45(38.8)$ \\
\hline PG diploma & $33(28.4)$ \\
\hline MCGP & 29(25) \\
\hline Other & $9(7.8)$ \\
\hline \multicolumn{2}{|l|}{ Education Specialty } \\
\hline Family medicine & $58(50)$ \\
\hline Community Medicine & $3(2.6)$ \\
\hline Other specialties & $5(4.3)$ \\
\hline Not specialized (Bachelor's degree only) & $50(43.1)$ \\
\hline \multicolumn{2}{|l|}{ Involvement in GP practice } \\
\hline Full time & $51(43.9)$ \\
\hline Part time & $65(56.1)$ \\
\hline \multicolumn{2}{|l|}{ Previous general surgical training } \\
\hline Internship & $53(45.7)$ \\
\hline Post internship & $39(33.6)$ \\
\hline Neither & $24(20.7)$ \\
\hline \multicolumn{2}{|l|}{ Distance to nearest CPD center ${ }^{1}$ (in km) } \\
\hline$<=25$ & $53(45.7)$ \\
\hline $26-50$ & $25(21.6)$ \\
\hline $51-100$ & 8(6.9) \\
\hline $101-150$ & 10(8.6) \\
\hline $151-200$ & $19(16.3)$ \\
\hline$>200$ & $1(0.9)$ \\
\hline \multicolumn{2}{|c|}{ Participation in previous CPD programs on management of breast carcinoma } \\
\hline Yes & $29(25.0)$ \\
\hline No & $87(75.0)$ \\
\hline \multicolumn{2}{|c|}{ Mode of delivery of previous CPD programs on management of breast carcinoma $(\mathrm{N}=29)$} \\
\hline Face to face & $29(100)$ \\
\hline \multicolumn{2}{|l|}{ Awareness on NCCP guide ${ }^{2}$} \\
\hline Aware and gone through & $20(17.2)$ \\
\hline Aware but not gone through & $11(9.5)$ \\
\hline Not aware & $85(73.3)$ \\
\hline \multicolumn{2}{|l|}{ Awareness on National Guidelines ${ }^{3}$} \\
\hline Aware and gone through & $8(6.9)$ \\
\hline Aware but not gone through & $12(10.3)$ \\
\hline Not aware & $96(82.8)$ \\
\hline
\end{tabular}

${ }^{1}$ Colombo, Kandy, Galle, Jaffna were considered as CPD centers.; ${ }^{2}$ Guide for primary care doctors and family physicians on management of breast symptoms published by National Cancer Control Program; ${ }^{3}$ National guidelines on breast cancer management. 
Table 2: Learning needs to be addressed in a future CPD module on management of breast carcinoma

\begin{tabular}{lcccccc}
\hline Learning need & \multicolumn{7}{c}{ Priority, $\mathbf{n}(\%)$} \\
\hline & $\begin{array}{c}\text { First } \\
\text { priority }\end{array}$ & $\begin{array}{c}\text { Second } \\
\text { priority }\end{array}$ & $\begin{array}{c}\text { Third } \\
\text { priority }\end{array}$ & $\begin{array}{c}\text { Fourth } \\
\text { priority }\end{array}$ & $\begin{array}{c}\text { Fifth } \\
\text { priority }\end{array}$ & $\begin{array}{c}\text { Sixth } \\
\text { priority }\end{array}$ \\
Palliative care & $\mathbf{3 9 ( 3 3 . 6 )}$ & $29(25.0)$ & $28(24.1)$ & $10(8.6)$ & $4(3.4)$ & $6(5.2)$ \\
Shared follow up care & $29(25.0)$ & $\mathbf{4 8 ( 4 1 . 4 )}$ & $26(22.4)$ & $6(5.2)$ & $3(2.6)$ & $4(3.4)$ \\
Treatment modalities & $16(13.8)$ & $19(16.4)$ & $\mathbf{2 6 ( 2 2 . 4 )}$ & $18(15.5)$ & $14(12.1)$ & $23(19.8)$ \\
Screening & $13(11.2)$ & $8(6.9)$ & $11(9.5)$ & $\mathbf{3 7 ( 3 1 . 9 )}$ & $18(15.5)$ & $29(25.0)$ \\
Referral for specialist care & $11(9.5)$ & $6(5.2)$ & $15(12.9)$ & $26(22.4)$ & $\mathbf{3 0 ( 2 5 . 9 )}$ & $28(24.1)$ \\
Health education & $8(6.9)$ & $6(5.2)$ & $10(8.6)$ & $19(16.4)$ & $\mathbf{4 7 ( 4 0 . 5 )}$ & $26(22.4)$ \\
\hline
\end{tabular}

Due to high levels of internet access and competence in using internet, CPD modules delivered online have been identified as the desired methods of acquiring these competencies in other countries (Smith et al., 2011) with comparable preferences among Sri Lankan GPs according to our study. This could be based on the better accessibility, flexible scheduling suited to individual requirements, frequent updates and the relative cost effectiveness when compared with traditional CPD training delivered face to face.

The study had to be limited to GPs affiliated to College of General Practitioners of Sri Lanka due to the unavailability of a comprehensive database of all GPs working in full time or part time basis throughout the country.

\section{Conclusion}

Participation of general practitioners in CPD programs on breast cancer management and awareness on current guidelines are deficient. Although GP's current involvement in referral of breast cancer patients for tertiary care is substantial, the involvement in shared follow up care and palliative care is markedly low. It is therefore recommended that a future online/blended CPD module should be established with greater emphasize on these two areas while also addressing the other components of breast cancer management.

\section{Acknowledgement}

Thanya I Pathirana was supported by the Fogarty International Centre, National Institutes of Health, under Award Number: D43TW008332 (ASCEND Research Network).
The contents of this publication is solely the responsibility of the authors and does not necessarily represent the official views of the National Institutes of Health or the ASCEND Research Network. We also wish to express our gratitude to the College of General Practitioners of Sri Lanka and very especially to all the general practitioners who participated in this study.

\section{References}

Epa, S. (2005) CPD Certification of Sri Lankan doctors, Ceylon Medical Journal, 50, 2, pp. 7678.

Grunfeld, E. (2008) Primary Care Physicians and Oncologists Are Players on the Same Team, Journal of Clinical Oncology, 26, 14, pp. 22462247.

Mitchell, G., Burridge, L., Colquist, S. \& Love, A (2012) General Practitioners' perceptions of their role in cancer care and factors which influence this role, Health \& Social Care in the Community, 20, 6, pp. 607-616.

National Cancer Control Programme (2012) Management of Breast Symptoms: Guide for primary care doctors and family physicians, Ministry of Health, Sri Lanka.

Roorda, C., De Bock, G., Van Der Veen, W., Lindeman, A., Jansen, L \& Van Der Meer, K. (2012) Role of the general practitioner during the active breast cancer treatment phase: an analysis of health care use, Supportive Care In Cancer: Official Journal Of The Multinational Association Of Supportive Care In Cancer, 20, 4, pp. 705-714.

Smith, S., Wai, E., Alexander, C. \& Singh-Carlson, S. (2011) Caring for survivors of breast cancer: perspective of the primary care physician, Current oncology, 18, 5, pp. 218. 